\title{
A rapid, maskless 3D prototyping for fabrication of capillary circuits:
}

\section{towards urinary protein detection}

Sheng Yan, ${ }^{a \dagger *}$ Yuanqing Zhu, ${ }^{\mathrm{b} \dagger}$ Shi-Yang Tang, ${ }^{a}$ Yuxing Li, ${ }^{a}$ Qianbin Zhao, ${ }^{a}$ Dan

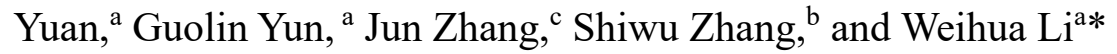

${ }^{a}$ School of Mechanical, Materials and Mechatronic Engineering, University of Wollongong, Wollongong, NSW 2522, Australia. E-mail: weihuali@uow.edu.au; sy034@uowmail.edu.au

${ }^{\mathrm{b}}$ Department of Precision Machinery and Instrumentation, University of Science and Technology of China, Hefei 230031, China

'School of Mechanical Engineering, Nanjing University of Science and Technology, Nanjing 210094, China.

${ }^{\dagger}$ The authors contribute equally.

Abbreviations: FDM: Fused Deposition Modeling; SLA: Stereolithography; POC: point-of-care; PMMA: Poly(methyl methacrylate); RBV: Retention Burst Valve; BSA: Bovine Serum Albumin

Keywords: Capillary microfluidics/ Rapid prototyping/ Laser ablation/ Urinary protein detection

Total number: 4991

\begin{abstract}
Proteinuria is an established risk marker for progressive renal function loss and patients would significantly benefit from a point-of-care testing. Although extensive work has been done to develop the microfluidic devices for the detection of urinary protein, they need the complicated operation and bulky peripherals. Here, we present a rapid, maskless 3D prototyping for fabrication of capillary fluidic circuits using laser engraving. The capillary circuits can be fabricated in a short amount of time $(<10 \mathrm{~min})$ without the requirements of clean-room facilities and photomasks. The advanced capillary components (e.g., trigger valves, retention valves and retention bursting valves) were fabricated, enabling the sequential liquid delivery and sample-reagent mixing. With the integration of smartphone-based detection platform, the microfluidic device can quantify the urinary protein via a colorimetric analysis. By eliminating the bulky and expensive equipment, this smartphone-based detection platform is portable for on-site quantitative detection.
\end{abstract}

\section{Introduction}

Proteinuria is a widely-recognized risk factor of renal diseases, as well as cardiovascular and organ damage. ${ }^{1}$ Especially, to treat many renal disorders, the accurate quantification of protein in urine is a fundamental requirement for diagnostic tools. ${ }^{2}$ Quantitation of proteinuria is considerably valuable in evaluating the therapy efficiency and the progression of disease. However, most advanced diagnostic technologies require highly trained staff and specialized facilities. ${ }^{3}$ 
There is a demand for an easy to use device that can detect protein in urine of clinical samples with less reagents and in a reduced amount of time. The microfluidic and lab-on-a-chip systems emerge, offering healthcare accessibility and affordability, quick results, timely management, and improved efficiency. ${ }^{4}$ Electrochemical sensing 5 and electrophoretic detection ${ }^{6-8}$ have been widely used for detection of urinary protein. However, these methods require bulky facilities (i.e., power supply, amplifier), which limit the devices' miniaturization and portability. To achieve this goal, capillary-driven microfluidic device is an attractive candidate due to its nature of self-powered and self-regulated completion of biochemical assays. ${ }^{9}$ Without the need of any external pumps and bulky peripheral equipment, the capillary microfluidic device is portable for on-site detection. Recently, an autonomous capillary microfluidic device was proposed to detect the urinary tract infection in a rapid and facile manner. ${ }^{10}$ This system provided a self-powered sequential liquid delivery for bacteria trapping, washing and staining. Plevniak et al. ${ }^{11}$ developed a capillary-driven auto-mixing platform for rapid diagnosis of anemia. The oxidizing agents were mixed with blood by capillary force and a colorimetric analysis was implemented on the mixture with the assistance of smartphone. Likewise, Zhang et al. ${ }^{12}$ reported a capillary chip for diagnosis of dengue fever (a debilitating tropical disease) from saliva. The reagent and saliva were transported by capillary driven, lateral flow from inlet to test strip (detection site).

3D microfluidic point-of-care (POC) devices can achieve more complex and multiplexed functions with the rapid development of 3D microfabrication techniques. To create $3 \mathrm{D}$ structures, a conventional method is to bond multiple molded layers, ${ }^{13}$ forming complex devices that perform a host of mechanical movements, chemical sensing, transduction and analysis. Another proven technique is 3D printing as it can easily and iteratively manufacture proof-of-concept microfluidic prototypes in a low-cost, facile and rapid manner. ${ }^{14-16}$ Most consumer-grade 3D-printers are usually based on fused deposition modeling (FDM) or stereolithography (SLA). As the FDM-based printers have limited resolution, the 3D model has larger feature with a minimum width $(\geq 0.4 \mathrm{~mm})$ and normally has holes on the surface. The SLA-based printers have high resolution, which can achieve the feature resolutions down to $\sim 20$ $\mu \mathrm{m}$. However, it takes several hours to manufacture a model in the order of centimeter. Meanwhile, such 3D printers need to operate in the clean-room environments to achieve the best fabrication performance. ${ }^{17}$ In addition, PDMS-based microfluidic replicas, containing more advanced fluidic elements, such as valves, mixers and pumps, cannot be directly bonded on the 3D-printed microchips due to the incompatibility of material. ${ }^{18}$

Hence we report a laser-ablated, maskless prototyping technique for the manufacture of geometry-based valves compatible with capillary-driven microfluidic devices. The devices are fabricated entirely from plain PDMS slabs which are widely used in capillary systems as PDMS is low-cost and has a good optical clarity for imaging purposes. Compared to the Poly(methyl methacrylate) (PMMA) which is well characterized for the formation of microfluidic channels using laser ablation-based techniques ${ }^{19}$, laser-engraved PDMS channel has smaller size under the 
same conditions and varies more linearly with the power and speed of the laser system. The prevalent conclusion is that the laser cutting created 'Gaussian like' channels ${ }^{19}$ with limited control over the channel dimension, thus preventing the integration of more advanced elements such as retention burst valves (RBVs), which are common components for advanced capillaric circuits. ${ }^{20}$ However, in present work, we first present the demonstration of laser-cutting RBVs formed within PDMS substrate. After integrating RBVs with other capillary fluidic elements within a capillaric circuit, we achieve autonomous delivery of liquids in a preprogrammed sequence according to the increasing order of RBV capillary pressure.

The capillaric circuits made by using $\mathrm{CO}_{2}$-laser-cutting can reduce fabrication cost, and do not require a clean-room environment and photomasks or embossing tools, and takes less than $10 \mathrm{~min}$ to produce a device. Moreover, we further demonstrate an application for diagnosis of proteinuria by mixing the urine with the dye reagent. The procedure consists of three steps: (1) introduce the sample into the reservoir, (2) introduce the reagent onto the injection port of release channel, and (3) mix the urine sample and reagent by diffusion. The assay results were quantified via a colorimetric analysis. The device was also integrated with a smartphone-based readout; and the whole assay was completed in $25 \mathrm{~min}$.

\section{Materials and methods}

\subsection{Principles of Capillaric Circuit}

As advanced capillaric circuits, the capillary valves including trigger valves, retention valves and RBVs and capillary pumps are required to achieve the versatile functions.

The trigger valves in the capillaric circuits play a dual role: (1) stopping liquids during initial filling of reservoirs,(2) acting as retention valves with higher capillary pressure than the capillary pump during reservoir drainage. ${ }^{20}$ These retention valves ensure that the side branches are not completely emptied, allowing sequential liquid delivery without bubble trapping. ${ }^{21}$

To stop the liquid in reservoir, an abrupt geometric change is normally designed in the cross section of microchannel to modify the capillary pressure at the junction, preventing the liquid flowing from the narrow side branch to the wider release channel. ${ }^{22}$ Briefly, the pressure change in capillary microfluidics can be derived by applying Young's equations to the total interfacial energy of the solid-liquid-air system and examining the changes due to a loaded liquid volume $V_{1},{ }^{19}$

$$
\mathrm{P}=-\frac{\mathrm{dU}_{\mathrm{T}}}{\mathrm{dV}_{\mathrm{l}}}=\gamma_{\mathrm{la}}\left(\cos \theta_{\mathrm{c}} \frac{\mathrm{dA}_{\mathrm{sl}}}{\mathrm{dV}_{\mathrm{l}}}-\frac{\mathrm{dA}_{\mathrm{la}}}{\mathrm{dV}_{\mathrm{l}}}\right)
$$

where $U_{\mathrm{T}}$ is the total interfacial energy of the system, $\gamma_{1 \mathrm{a}}$ is the surface tension at the air-liquid interface, $\theta_{\mathrm{c}}$ is the contact angle, $A s 1$ is the area of the wetted surface and $A_{1 \mathrm{a}}$ is the area of the liquid-air interface. For constant microchannels, the capillary pressure will stay positive. As $A_{S 1}$ remains fixed, the pressure becomes negative and stops the liquid with the increase of air- liquid surface area caused by an abrupt geometric change in the microchannel. The trigger valves can then be triggered by a sample flowing through the release channel.

$\mathrm{RBV}$ is a modification of the retention valve, and both are formed by a localized 
reduction of the channel junction preventing the liquid from draining due to a large capillary pressure. They work with the capillary pumps to achieve a predetermined sequence of drain. Capillary pumps generate constant capillary pressure providing power in capillary circuit. The capillary pressure is related to the smallest dimension in cross-section of the capillary pumps. The smaller the dimension is, the larger the pressure drop and the stronger the pump will be generated. ${ }^{23}$

RBVs will hold the liquid until the pressure at a point within the circuit drops below the capacity of the valve. ${ }^{21}$ When the fluid flows in the circuit, the pressure drop across the microchannel makes the hydrodynamic pressure of any point in the vicinity of the RBV weaker than the receding capillary pressure of the RBV. As a result, the liquid stops. Once the drain stops, the circuit turns to a hydraulic system and the capillary pressure of the pump is transmitted throughout, causing pressure of any point in the vicinity of the RBV stronger than the receding capillary pressure of the RBV. Eventually, the RBV bursts. ${ }^{21}$ The threshold of RBV is related to its physical geometry. The smaller the geometry of the RBV is, the larger the burst pressure of it will be. For the capillaric circuits with multiple retention burst valves having different thresholds, the weakest one will burst first draining the liquid stored in the reservoir downstream, and then the second weakest one will burst draining the liquid.

\subsection{Laser-based Manufacturing and Device Characterization}

All microfluidic channels utilized in present work were fabricated on PDMS slab using a $\mathrm{CO}_{2}$ laser system (ILS9.75, Universal Laser Systems, Inc., USA). The laser system has a fixed working area where PDMS slabs are placed on the working stage. The stage can be adjusted in the $z$-direction to focus the laser beam with different height of the samples. The laser is directed along the processed base by way of light guiding and focusing lenses that are moveable in the $x$ and $y$ directions by 2 DC servomotors, with a maximum resolution of 1000 PPI over a total working area of $914 \times 610 \mathrm{~mm}^{2}$. The laser system has a maximum power of $25 \mathrm{~W}$ working at a wavelength of $10.6 \mu \mathrm{m}$. Designs of the microfluidic channel were made using AutoCAD (Autodesk, Inc., USA), which was interfaced directly with the $\mathrm{CO}_{2}$ laser-cutting machine so that designs could be etched directly onto a target base. The scan speed and power of the laser can be modified before etching to achieve the desired depth of microchannel and is controllable covering a percentage scale of 0 $100 \%$, in $1 \%$ increment. Before using, several speed and power settings were tested to determine the corresponding depth of microchannel systems.

To make PDMS base, elastomer base and curing agent (sylgard ${ }^{\circledR} 184$, Dow Coming Corporation, USA) were mixed in a 10: 1 ratio. The PDMS mixture was degassed and then poured into a smooth flat dish. Then PDMS was cured for $2 \mathrm{~h}$ at $90{ }^{\circ} \mathrm{C}$. After laser engraving, PDMS slabs were cleaned by isopropanol (Sigma-Aldrich, USA) to remove the surface carbonized tissue, then activated for 180 seconds at 200 mTorr and 45W RF power in a plasma chamber (PDC-001-HP, Harrick Plasma, USA). The treated PDMS devices were covered by a flat, untreated PDMS plate to provide closed microchannels for capillary-driven flow. Flow in capillaric circuits was tested using aqueous food dye and recorded by a video camera 
(DP-M01, Oriental Inspiration Limited, Hong Kong).

\subsection{Protein Assay}

The protein assay reagent used in present work was purchased from Bio-Rad. The basis of reagent to react with protein is Coomassie Brilliant blue G-250 dye, which has the absorption peak shifting from $465 \mathrm{~nm}$ to $595 \mathrm{~nm}$. By determining the absorbance, the protein concentration can be quantified with a calibration curve. ${ }^{18}$ The reagent was diluted to $1 / 5.5$ of the original concentrate by distilled water prior to experiment. During the experiment, sample and reagent react in a ratio of 1:9.

Artificial urine spiked with bovine serum albumin (BSA, Sigma-Aldrich, USA) was used as the model sample solution. The artificial urine was prepared following previous works. ${ }^{18}$ It contained $1.1 \mathrm{mM}$ lactic acid, $2.0 \mathrm{mM}$ citric acid, $25 \mathrm{mM}$ sodium bicarbonate, $170 \mathrm{mM}$ urea, $2.5 \mathrm{mM}$ calcium chloride, $90 \mathrm{mM}$ sodium chloride, 2.0 $\mathrm{mM}$ magnesium sulfate, $10 \mathrm{mM}$ sodium sulfate, $7.0 \mathrm{mM}$ potassium dihydrogen phosphate, $7.0 \mathrm{mM}$ dipotassium hydrogen phosphate, and $25 \mathrm{mM}$ ammonium chloride. The final $\mathrm{pH}$ was adjusted to 6.0 by addition of hydrochloric acid. BSA was used to represent the protein sample since albumin is the most abundant urinary protein in patients with proteinuria. ${ }^{24}$

\subsection{Smartphone-Based Quantification and Image Acquisition}

Diagnostic technology typically requires image to quantify the amount of sample. The smartphone is a good platform for diagnosis due to its easy imaging, processing and communication capability, and popular using. ${ }^{18}$ The smartphone-based diagnostic platform consists of a breadboard with 4 LEDs (590nm emission), a battery and a resistor, light diffuser, 3D-printed holders and a smartphone. A Meizu PRO 5 (with a 21.16 megapixel camera) was used as the model smartphone in this work. The images were taken by the native camera application in which the exposure time and photosensibility can be fixed. Then the images were sent to a computer for further processing. As the light source was chosen to match the absorption of the reagent, the light intensity in the region of interest was the only information required to obtain the absorbance. Thus, the acquired colored image was converted to 8-bit gray scale format to transfer the raw three RGB (Red, Green, and Blue) channel data into a single gray channel light intensity data by ImageJ. After extracting the light intensity data, absorbance was calculated with the formula $A b s=-\log \left(I_{1} / I_{0}\right)$, where $I_{1}$ is the average light intensity of the reaction chamber and $\mathrm{I}_{0}$ is that of the reference region.

\section{Results and Discussion}

\subsection{Characterization of Laser Engraving}

Firstly, to demonstrate the 3D prototyping using laser engraving, the investigation was conducted on the parameters including cutting speed, power, as well as different materials. Fig. 1 showed the channel depth and width of PDMS and PMMA with the different laser bean moving speeds and powers. Compared to PMMA at a cutting speed of $80 \%$ of the full speed, PDMS has smaller channel in cross-section after engraved. Besides, the channel width on the PDMS varied more linearly with the power. Therefore, the size of the microfluidic channel on PDMS base is much more 
controllable than that on PMMA substrate. The height and width of the PDMS channel increased when applying a larger laser power or decreasing the laser moving speed, as either higher power or lower moving speed can make PDMS accumulate more heat to carbonize. Noticeably, when the power is larger than $50 \%$ and the speed is smaller than $60 \%$, the height change of the channel tends to be saturated due to the severe carbonization of the surface.

Normally, the single pass of laser can generate the channel with a width around $200 \mu \mathrm{m}$. However, to form wider channels (larger than $200 \mu \mathrm{m}$ ), multiple passes of the laser are required, and results in more rectangular-shaped channels. The multiple scans of the laser and the Gaussian distribution of the power can form 'trench' structure on the PDMS (See supporting information Fig. S1). From previous testing, for PMMA cutting, flow characteristics resulting from defects were found to be negligible for microchannel widths larger than $200 \mu \mathrm{m}$ and heights larger than 100 $\mu \mathrm{m} .{ }^{19}$ Considering that the PDMS has smaller defects than PMMA, these dimensions are still valid for PDMS channel. These dimensions therefore set the minimal limit to the channel size in our experiments. In our experiments, minimum microchannels (trigger valve) with the widths of $230 \mu \mathrm{m}$ and heights of $300 \mu \mathrm{m}$ were fabricated to demonstrate that the effect of defects is negligible during fluidic flowing. 

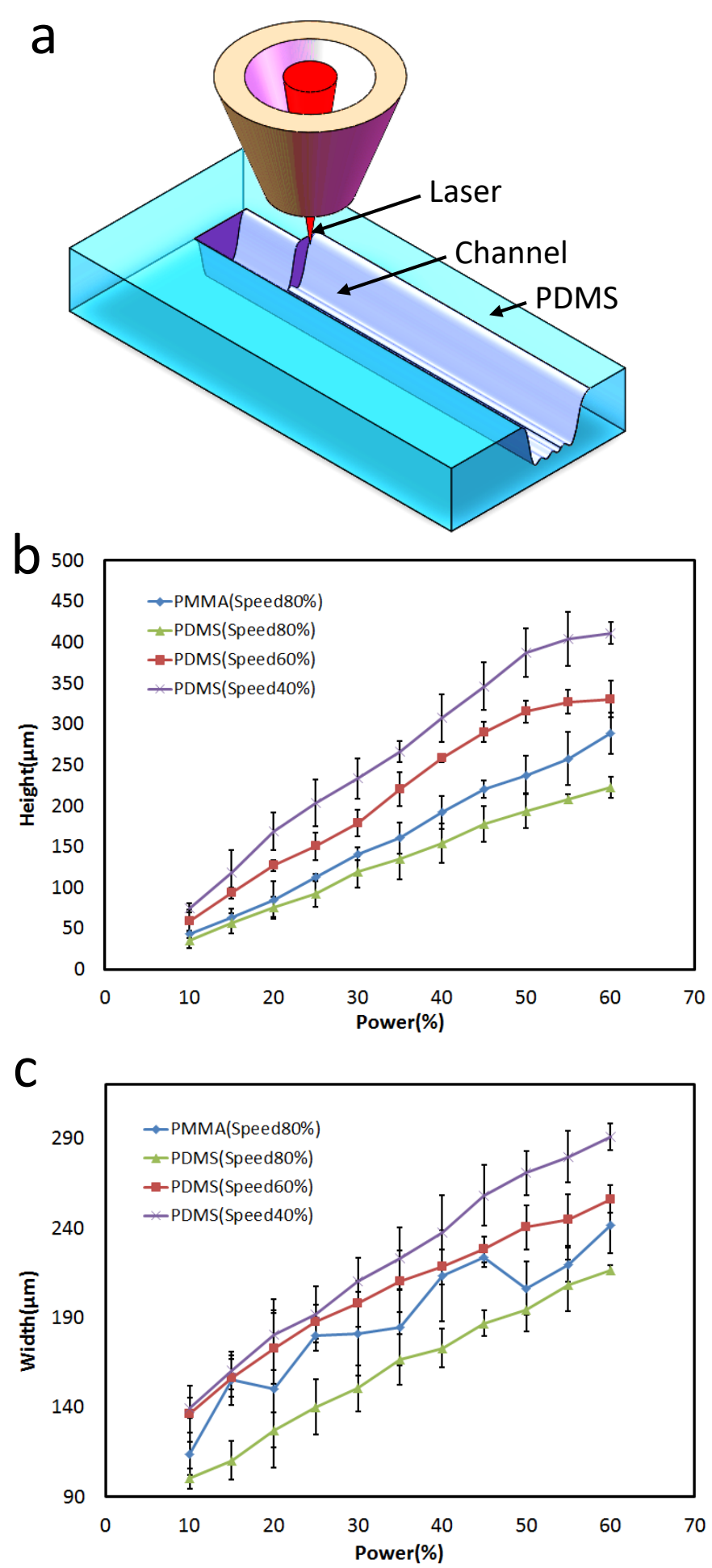

Figure 1 Comparison with the geometry between PMMA and PDMS. a) Schematic showing maskless 3D prototyping using laser engraving. b) Height for different power and speed of laser treating. c) Width for different power and speed of laser treating. The average value was 3 times the measurement and the error bar represents standard deviation.

\subsection{Success Rate of trigger valves}

Here we defined trigger valve success as when a valve holds liquid for at least 10 
minutes without leakage. The success rate of trigger valves is highly related to their geometries. $^{25}$ The geometric parameters known to affect the performance of capillary trigger valves are the height and width of the trigger valve, and the height difference between the TV and the release channel (Fig. S2a). ${ }^{20}$ In this work, the height of the trigger valve was related to the width due to the 'Gauss like' characteristics of the microchannel, so we tested the height differences between the trigger valves and the release channel. The release channels used for experiments were fixed to be $340 \mu \mathrm{m}$ in depth. In the actual manufacturing process, to ensure that the release channel and trigger valves are fully connected, the redundant processing (length $\lambda$ in Fig. S2b) is needed. Given that the surface property will not change after laser engraving, the height difference $\Delta h$ is equal to the high of the release channel (Fig. S2b). The success rate corresponding to the height difference was then investigated (Fig. S2c). The height difference between the trigger valve and the release channel had a threshold effect on success rate of trigger valve. The success rate was about $50 \%$ without height difference, whereas the success rate reached nearly $100 \%(N=10)$ when the height difference was over $200 \mu \mathrm{m}$. Since the release channel is always larger than $300 \mu \mathrm{m}$ in our experiments, the trigger valves will have a nearly $100 \%$ success rate during the actual operation.

\subsection{Pre-programmed Capillary Microfluidics for Sequential Fluid Delivery}

To demonstrate the maskless $3 \mathrm{D}$ prototyping technique for fabrication of capillaric circuits with pre-programmed liquid drain sequence, we designed a circuit with 5 RBVs (Fig. 2a, b). Different from cleanroom-fabricated devices in which the width is the only free parameter to adjust the threshold of RBV, the capillaric circuits designed in this work can adjust width and height independently by multiple engraving of the laser. The smallest reservoirs were $710 \mu \mathrm{m}$ wide, $560 \mu \mathrm{m}$ deep, and $5000 \mu \mathrm{m}$ long with a volume of $\sim 1.6 \mu \mathrm{L}$, and the largest reservoir was $760 \mu \mathrm{m}$ wide, $590 \mu \mathrm{m}$ deep, and $8000 \mu \mathrm{m}$ long with a volume of $\sim 2.75 \mu \mathrm{L}$, and the release channel was $530 \mu \mathrm{m}$ wide, $560 \mu \mathrm{m}$ high, and $11000 \mu \mathrm{m}$ long with a volume of $1.64 \mu \mathrm{L}$. The geometries of the RBVs in the capillaric circuits are summarized in Table S1.

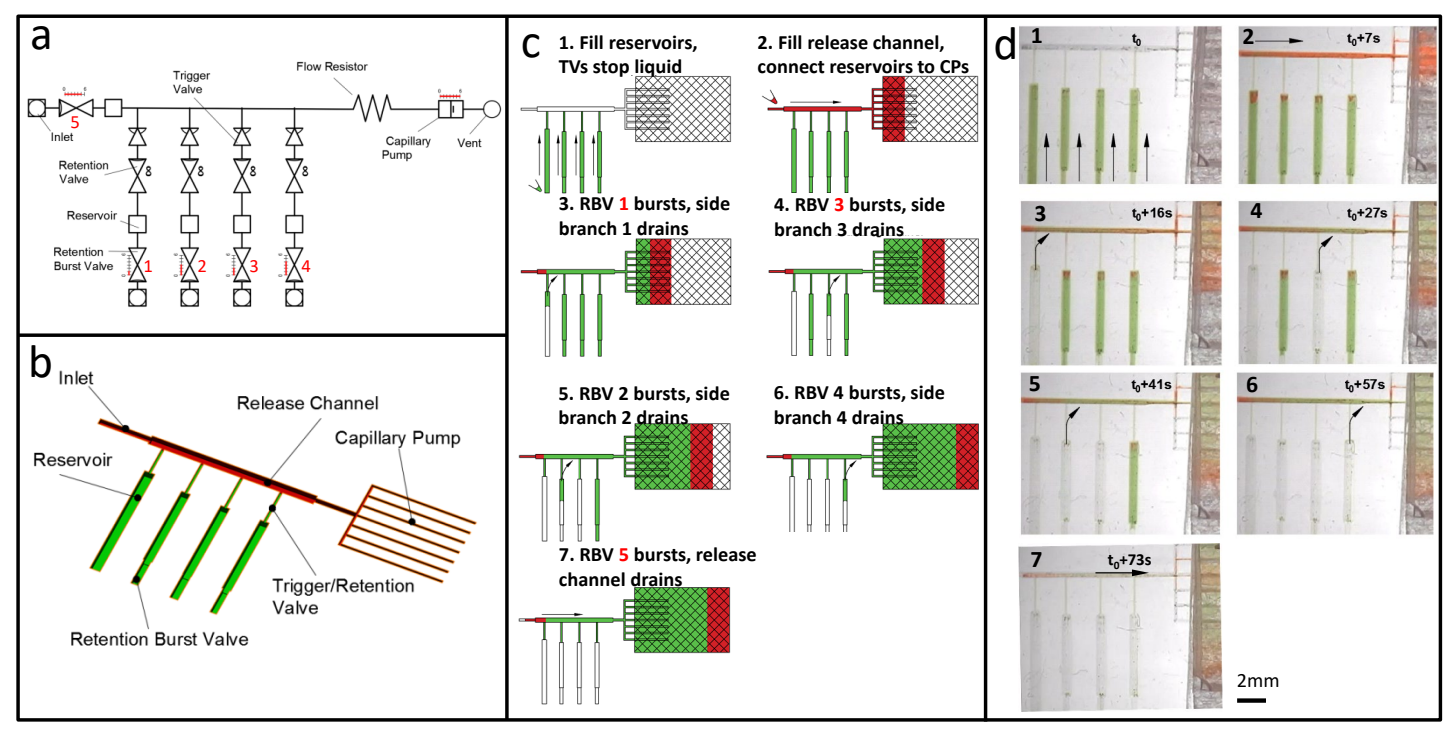


Figure 2 Design, mold, operation and time-lapse images of capillaric circuits for autonomous sequential delivery of five liquids. a) Symbolic representation of capillaric circuit with main fluidic elements labeled. b) Schematic of capillaric circuit. c) Schematic illustrating expected operation of RBVs. Solutions loaded into the reservoirs are delivered in pre-programmed manner according to RBV capillary pressure. d) Time-lapse images showing autonomous and sequential drainage of reservoirs in the capillaric circuit. Arrows represent sequence and flow direction. Text labels show time during liquid delivery. A video of the autonomous liquid delivery operation is provided in movie $\mathrm{S} 1$.

The expected pre-programmed drain of the capillaric circuit is shown in Fig. 2c. Since the larger RBV has lower burst pressure, the designed delivery sequence is: $\mathrm{RBV} 1 \rightarrow \mathrm{RBV} 3 \rightarrow \mathrm{RBV} 2 \rightarrow \mathrm{RBV} 4 \rightarrow \mathrm{RBV} 5$. We tested liquid delivery using aqueous food dye. Firstly, reservoirs were initially pre-filled and trigger valves held green-dye liquids. Next, a red-dye solution was added to the inlet of release channel, connecting the reservoirs to the pump. Then, a piece of cleanroom paper was put on the capillary pump. Once the solution from inlet of the release channel touched the cleanroom paper, the capillary pump started functioning, which gradually increased the negative pressure inside the channel. RBV1 firstly finished the drain at $t=t_{0}+16 \mathrm{~s}$, following by RBV3, RBV2, RBV4 and RBV5 (Fig. 2d). As designed, each side branch drained sequentially without drainage of the other RBVs. Each reservoir take 9 16 s to drain and the autonomous drainage of the 4 side branches was completed in $<1 \mathrm{~min}$. The average flow rates for liquid drainage from branches were shown in Fig. S3. The average flow rate is calculated by dividing the liquid volume of branch by the drainage time.

\subsection{Capillary Microfluidics for Liquid Mixing}

To demonstrate the capability of mixing using the capillaric circuits fabricated by maskless prototyping technique, we designed a circuit with 3 side branches, shown in Fig. 3. The hydrophilic PDMS bases are covered by untreated hydrophobic PDMS plates. We also tested mixing using aqueous food dye. Green liquids were initially pre-filled into the trenches of the reservoirs (See supporting information Fig. S4). If overfilled, these liquids would be hold by the retention valves. After the main channel was filled with red liquid, the red-dye solution entered the reservoirs through retention valves to mix with green medium. It normally takes $8 \mathrm{~s}$ to fill the reservoir up. If there was no liquid in reservoirs, the retention valves played the same role for the main channel due to the height difference between main channel and side branch, which can prevent the liquid entering the reservoirs. Since the green-dye solutions fill the trenches located at the bottom of reservoirs and red-dye solution fill the rest of the reservoirs, the ratio between the two liquids can be controlled by the volumes of trenches, which can be adjusted by varying distances between each engraving of the laser, shown in Table S2. With the increase of distance between each engraving, the volume of green solution in the trench will increase and the ratio of green solution to red solution will be changed. Due to the carbonized surface that has adverse effect on the PDMS transparency, the present device is not suitable for quantitative analysis. Nonetheless, this device can be used to qualitative analysis of the sample, especially for those assays with obvious color change, such as $\mathrm{pH}$ testing. 


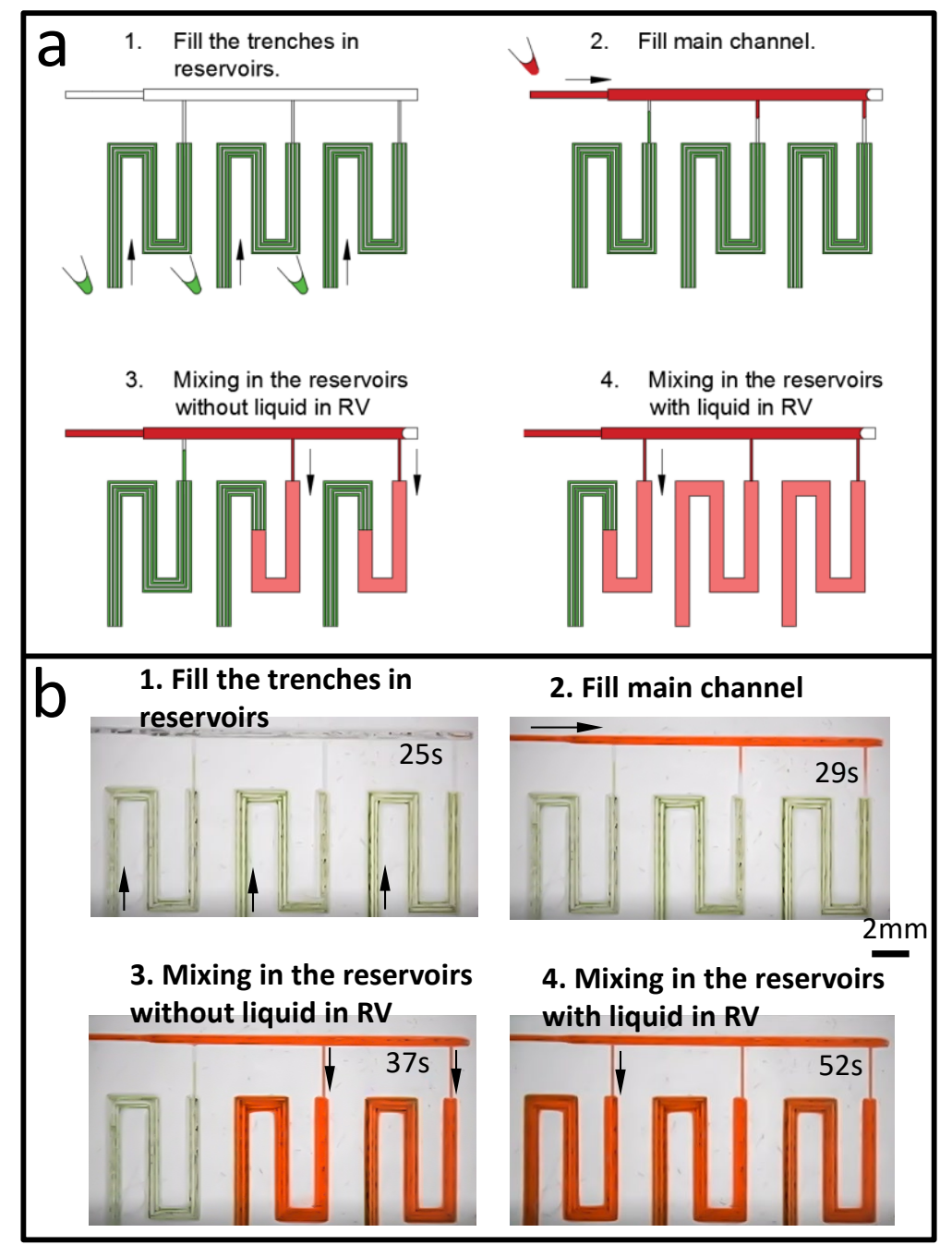

Figure 3 Operation and time-lapse images of capillaric circuits for mixing of aqueous food dyes. a) Schematic illustrating expected operation of mixing process. b) Time-lapse images showing mixing in the reservoirs of capillaric circuit. Arrows represent sequence and flow direction. Text labels show time during operation. A video of the liquid operation is provided in movie S2.

\subsection{Smartphone-based Platform for Urinary Protein Quantification}

To overcome the limitation in the mixing chip described above, the chip is further modified by punching holes on the PDMS slabs ( $3 \mathrm{~mm}$ thick) and bonding to glass slides to form reservoirs (Fig. 4). In contrast to PDMS trenches, the reservoir with smooth, transparent glasses can effectively reduce interference from random noise caused by the carbonized surface. Also, a deeper reservoir enhances the contrast of images taken after absorbance.

The quantitative analysis of urinary protein was conducted via smartphone readouts (Fig. 5). The core components of testing device are shown in Fig. 5. Inside the core component, 4 LEDs with $590 \mathrm{~nm}$ emission were connected to a battery and a resistor. To gain uniform illumination of the chip, the top of LEDs and inner wall of the component were covered by tin foil to diffuse reflect the light, and a light diffuser was used to cover the diffuser holder. When imaging, the chip was placed upon the light diffuser, and a smartphone holder was located above the chip, which can fix the 
smartphone at the right place to focus. The LEDs were chosen to match the wavelength of absorption peak $\left(\lambda_{\max }=595 \mathrm{~nm}\right)$, meanwhile reduced interference from ambient light and enhanced detection sensitivity. To eliminate the interference caused by ambient light, a shield was used to cover the exit of the holder.

In this experiment, the reagent was diluted to $1 / 5.5$ of the original concentrate by distilled water. After image acquisition and processing, the linear range for quantification of urinary protein is from 20 to $200 \mu \mathrm{g} / \mathrm{mL}$ (Fig. 5d). To test the efficacy of device for urinary protein quantification, four samples with different urine protein concentrations $(0,20,90$, and $170 \mu \mathrm{g} / \mathrm{mL})$ as unknown samples were introduced to the reservoirs. The main channel was then filled with a diluted reagent (Fig. 4b). It normally takes $2 \mathrm{~min}$ to fill up all the reservoirs. The samples will not leak from reservoirs due to the effectiveness of retention valves. After $25 \mathrm{~min}$ incubation, the sample and the reagent were fully mixed and the whole chip was placed on smartphone-based platform to quantify the urinary protein. The absorbance of reacted samples can be measured by image processing and then compared with the standard curve (Fig. 5d). The measured results shown in the Table S3 were very close to the reference values.

Unlike the commercialized test strips that are designed to give qualitative (e.g., pregnancy test strips) or semiquantitative (e.g., $\mathrm{pH}$ indicators and urine test strips) results, the proposed platform can provide an accurate quantitative analysis, which is more informative. Multiple patients' samples can be tested at one time in our device and each retention valve can hold the urine sample to prevent the interference from other samples. More reservoirs can be fabricated to accommodate and test more samples at the same device. The linear range can be adjusted by diluting the reagent to test different renal diseases such as nephrotic syndrome, clinical albuminuria, and microalbuminuria ${ }^{24}$.

The capillaric circuits are driven by the capillary force, which can eliminate the need for bulky equipment, such as power supplies, syringe pumps and pressure units. Besides, the accessories of the smartphone-based platform are light and cheap. The most expensive parts, LED lights, cost about AU\$6. Based on these advancements, this device is portable for on-site quantification detection of the total protein content in urine.

In the future research, a dedicated smartphone application can be programmed in practical situations to process the image by smartphone and thus eliminate the needs of an external computer for image analysis. Besides, a smartphone-powered mixer using vibration of the motor will be integrated to enhance the mixing and shorten the mixing time. 


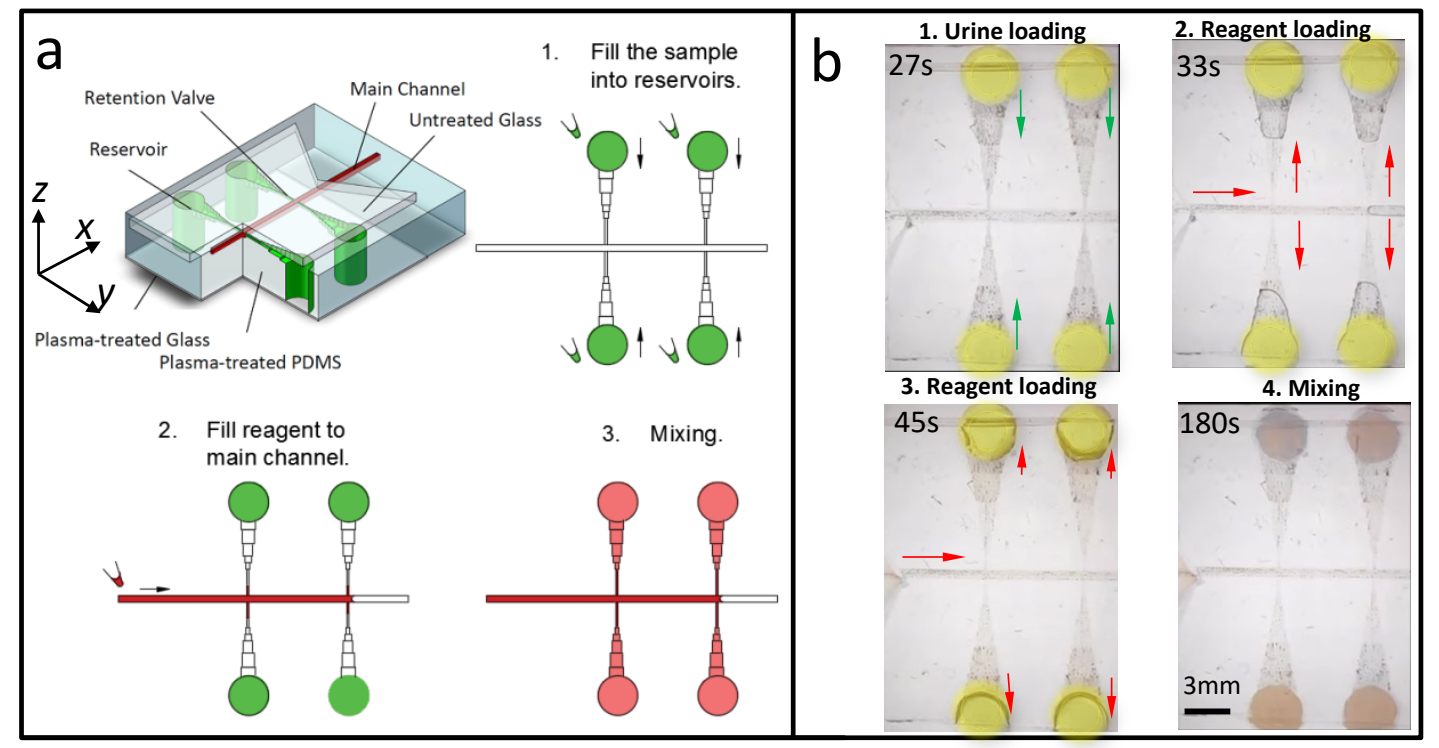

Figure 4 Operation and time-lapse images of capillaric circuits for mixing of sample and reagent. a) Schematic illustrating expected operation of mixing process. b) Time-lapse images showing mixing in the reservoirs of capillaric circuit. Arrows represent sequence and flow direction. Text labels show time during operation. A video of the liquid operation is provided in movie S3. 


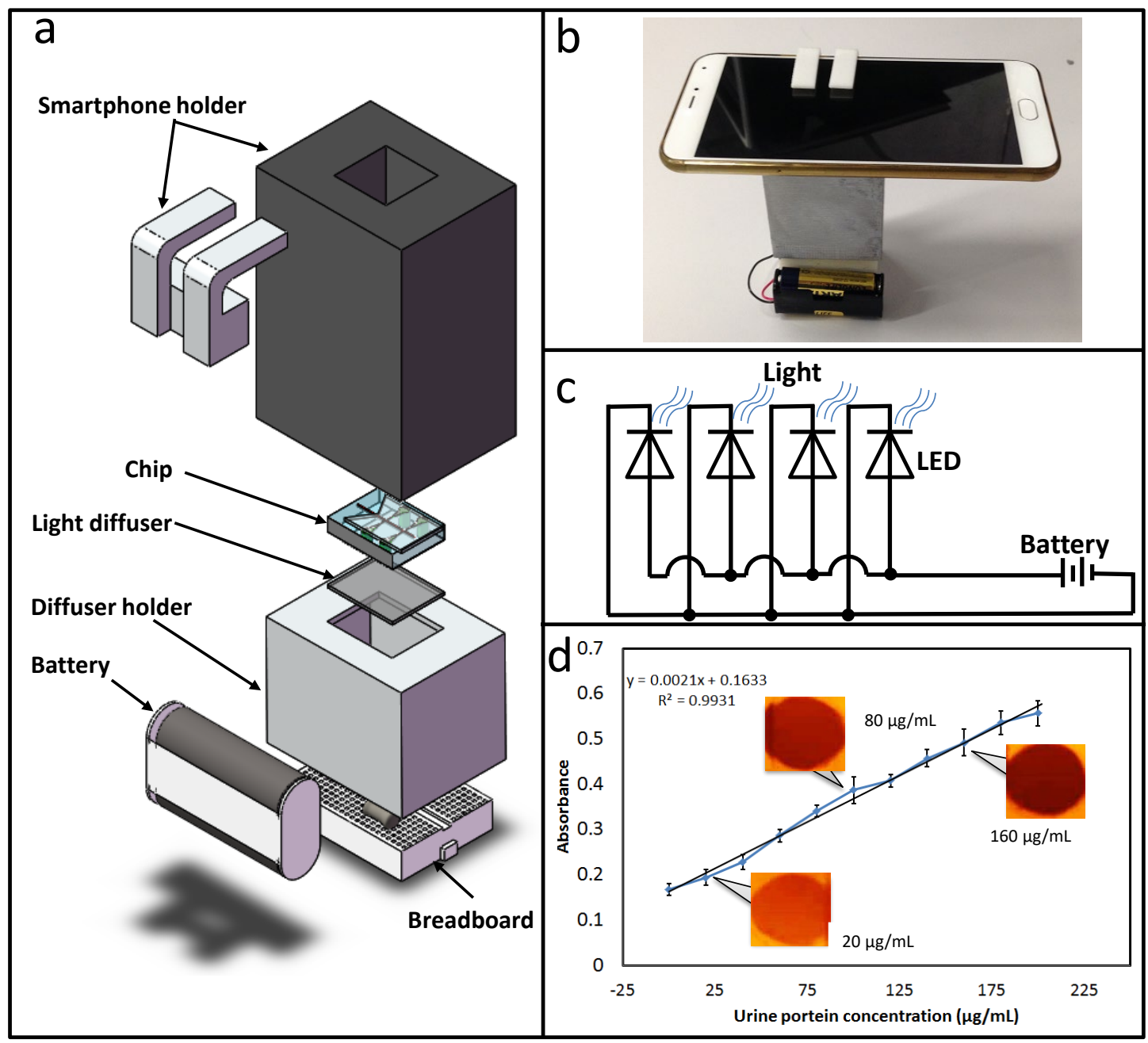

Figure 5 Schematic representation of the integrated chip for urinary protein quantification. a) The exploded view of the mold of smartphone-based detection platform. b) The photographic image of smartphone-based detection platform for quantifying total protein amount in the sample. The smartphone holder is used to fix smartphone and block ambient light. c) Schematic diagram of circuit of light source on the breadboard. d) Linear range of diluted reagent for quantification of urinary protein $(N=10$, error bar $= \pm$ standard deviation). (Inset) The optical image of the reservoir mixed with sample with different concentrations of protein.

\section{Concluding remarks}

In this work, we developed a rapid, maskless 3D prototyping for fabrication of capillaric circuits. The advanced capillary components including trigger valves, retention valves, RBVs and capillary pumps were fabricated by $\mathrm{CO}_{2}$ laser engraving without clean-room environment and photomasks. The device can be fabricated from design to use within $10 \mathrm{~min}$. The developed 3D capillaric circuits can achieve sequential liquid delivery and sample mixing. Together with the smartphone-based detection platform, the developed 3D capillaric circuits have the advantages of reduced reagent consumption, on-site quantitative detection, and low manufacturing cost (each chip for protein assay cost less than AU\$1). Multiple urine samples can be detected simultaneously at the same device within $30 \mathrm{~min}$. This portable 
smartphone-based detection platform is useful in diagnosis without the bulky and expensive equipment.

J.Z thanks the support from the Natural Science Foundation of Jiangsu Province (BK20170839) and the National Natural Science Foundation of China (Grant No.51705257).

The authors have declared no conflict of interest.

\section{References}

1. M. J. Sarnak, A. S. Levey, A. C. Schoolwerth, J. Coresh, B. Culleton, L. L. Hamm, P. A. McCullough, B. L. Kasiske, E. Kelepouris and M. J. Klag, Circulation, 2003, 108, 2154-2169.

2. P. Ruggenenti, A. Perna, L. Mosconi, R. Pisoni and G. Remuzzi, Kidney international, 1998, 53, 1209-1216.

3. R. W. Peeling and D. Mabey, Clinical Microbiology and Infection, 2010, 16, 1062-1069.

4. P. Novo, F. Volpetti, V. Chu and J. P. Conde, Lab on a chip, 2013, 13, 641-645.

5. C. Y. Liu, J. Rick, T. C. Chou, H. H. Lee and G. B. Lee, Biomed. Microdevices, 2009, 11, 201-211.

6. Y. Ni, J. Gan and Y. Xu, Proceedings of the Institution of Mechanical Engineers Part N Journal of Nanoengineering \& Nanosystems, 2014, 228, 57-60.

7. F. Li, R. M. Guijt and M. C. Breadmore, Anal. Chem., 2016, 88.

8. W. Ruige and Y. S. Fung, Bioanalysis, 2015, 7, 907-922.

9. L. Gervais, M. Hitzbleck and E. Delamarche, Biosens. Bioelectron., 2011, 27, 64-70.

10. A. O. Olanrewaju, A. Ng, P. DeCorwin-Martin, A. Robillard and D. Juncker, Anal. Chem., 2017.

11. K. Plevniak, M. Campbell, T. Myers, A. Hodges and M. He, Biomicrofluid., 2016, 10, 054113.

12. Y. Zhang, J. Bai and J. Y. Ying, Lab Chip, 2015, 15, 1465-1471.

13. D. P. Parekh, C. Ladd, L. Panich, K. Moussa and M. D. Dickey, Lab Chip, 2016, 16, 1812-1820.

14. N. Bhattacharjee, A. Urrios, S. Kang and A. Folch, Lab on a chip, 2016, 16, 1720-1742.

15. S. Waheed, J. M. Cabot, N. P. Macdonald, T. Lewis, R. M. Guijt, B. Paull and M. C. Breadmore, Lab on a chip, 2016, 16, 1993-2013.

16. C. M. Ho, S. H. Ng, K. H. Li and Y. J. Yoon, Lab on a chip, 2015, 15, 3627-3637.

17. V. A. Lifton, G. Lifton and S. Simon, Rapid Prototyping Journal, 2014, 20, 403 - 412.

18. H. N. Chan, Y. Shu, B. Xiong, Y. Chen, Y. Chen, Q. Tian, S. A. Michael, B. Shen and H. Wu, ACS Sensors, 2016, 1, 227-234.

19. M. I. Mohammed, E. Abraham and M. P. Y. Desmulliez, Journal of Micromechanics and Microengineering, 2013, 23, 035034.

20. A. O. Olanrewaju, A. Robillard, M. Dagher and D. Juncker, Lab on a chip, 2016, 16, 3804-3814.

21. R. Safavieh and D. Juncker, Lab on a chip, 2013, 13, 4180-4189.

22. M. Zimmermann, P. Hunziker and E. Delamarche, Microfluidics and Nanofluidics, 2008, 5, 395-402.

23. M. Zimmermann, H. Schmid, P. Hunziker and E. Delamarche, Lab on a chip, 2007, 7, 119-125.

24. A. W. Martinez, S. T. Phillips, E. Carrilho, S. W. Thomas III, H. Sindi and G. M. Whitesides, Anal. Chem., 2008, 80, 3699-3707.

25. K. Kistrup, C. E. Poulsen, P. F. Østergaard, K. B. Haugshøj, R. Taboryski, A. Wolff and M. F. Hansen, Journal of Micromechanics and Microengineering, 2014, 24, 125007. 